\title{
(6) OPEN ACCESS \\ Variation in use and outcomes related to midline catheters: results from a multicentre pilot study
}

\author{
Vineet Chopra, ${ }^{\oplus, 2,3}$ Scott Kaatz, ${ }^{4}$ Lakshmi Swaminathan, ${ }^{5}$ \\ Tanya Boldenow, ${ }^{6}$ Ashley Snyder, ${ }^{1,2,3}$ Rachel Burris, ${ }^{1,3}$ \\ Steve J Bernstein, ${ }^{2,3,7}$ Scott Flanders ${ }^{1,3}$
}

- Additional material is published online only. To view please visit the journal online (http://dx.doi.org/10.1136/ bmjas-2018-008554).

For numbered affiliations see end of article.

\section{Correspondence to}

Dr Vineet Chopra, Division of Hospital Medicine, University of Michigan, Ann Arbor, MI 48109, USA; vineetc@umich.edu

Received 6 July 2018 Revised 7 February 2019 Accepted 25 February 2019 Published Online First 18 March 2019
Check for updates

(C) Author(s) (or their employer(s)) 2019. Re-use permitted under CC BY-NC. No commercial re-use. See rights and permissions. Published by BMJ.

To cite: Chopra V, Kaatz S, Swaminathan $\mathrm{L}$, et al. BMJ Qual Saf

2019:28:714-720.

\begin{abstract}
Background While midline vascular catheters are gaining popularity in clinical practice, patterns of use and outcomes related to these devices are not well known. Methods Trained abstractors collected data from medical records of hospitalised patients who received midline catheters in 12 hospitals. Device characteristics, patterns of use and outcomes were assessed at device removal or at 30 days. Rates of major (upper-extremity deep vein thrombosis [DVT], bloodstream infection [BSI] and catheter occlusion) and minor complications were assessed. $\chi^{2}$ tests were used to examine differences in rates of complication by number of lumens, reasons for catheter removal I, and hospital-level differences in rates of midline use

Results Complete data on 1161 midlines representing $5 \%-72 \%$ of all midlines placed in participating hospitals between 1 January 2017 and 1 March 2018 were available. Most (70.8\%) midlines were placed in general ward settings for difficult intravenous access (61.4\%). The median dwell time of midlines across hospitals was 6 days; almost half (49\%) were removed within 5 days of insertion. A major or minor complication occurred in $10.3 \%$ of midlines, with minor complications such as dislodgement, leaking and infiltration accounting for $71 \%$ of all adverse events. While rates of major complications including occlusion, upper-extremity DVT and BSI were low $(2.2 \%, 1.4 \%$ and $0.3 \%$, respectively), they were just as likely to lead to midline removal as minor complications ( $53.8 \%$ vs $52.5 \%, p=0.90$ ). Across hospitals, absolute volume of midlines placed varied from 100 to 1837 devices, with corresponding utilisation rates of $0.97 \%-12.92 \%(p<0.001)$.

Conclusion Midline use and outcomes vary widely across hospitals. Although rates of major complications are low, device removal as a result of adverse events is common.
\end{abstract}

\section{INTRODUCTION}

First introduced in the 1950s, midline catheters are unique vascular access devices often referred to as 'middle ground' intravenous catheters. ' Like peripherally inserted central catheters (PICCs), midlines are inserted in the peripheral veins of the upper extremity; however, unlike PICCs, midlines terminate in the peripheral, not the central veins. By definition, the tip of the midline catheter should be located at or near the level of the axilla, distal to the shoulder. ${ }^{2}$ Because they are not central venous catheters, midlines cannot lead to central line-associated bloodstream infection (CLABSI), resulting in many hospitals preferentially using to these devices to avoid CLABSI and associated financial penalties. ${ }^{3}$ Additionally, as midlines are longer than traditional peripheral intravenous catheters and reach the deeper veins of the arm, they are potentially able to dwell longer than standard peripheral intravenous catheters. For these reasons, they offer a convenient alternative to PICCs for certain indications. ${ }^{5}$

With growing reports regarding overuse of PICCs and the risk of potentially avoidable complications, ${ }^{6}{ }^{7}$ renewed interest in the use of midlines has emerged. However, midline devices are not homogeneous. Rather, they vary in composition (eg, polyurethane vs silicone), configuration (single vs dual lumen), gauge (4 and 5 French) and insertion technique. Consequently, substantial variation regarding safety and outcomes of midlines exists. For example, in a single-centre retrospective study spanning 1538 midlines, dwell times ranged from 12 to 27 days (median 26) and occlusion was a common complication (1.44 events per 1000 midline days). ${ }^{8}$ In another study comparing outcomes between PICCs and midlines, patients who received midlines were observed to experience more complications than those with PICCs $(19.5 \%$ vs $5.8 \%, \mathrm{p}<0.001)$, with no difference in rates of severe complications. ${ }^{9}$ In contrast, a third quasi-experimental study found that introduction of appropriateness criteria to guide the 
selection of midlines versus PICCs led to a reduction in hospital PICC use, rates of upper-extremity thrombosis and catheter occlusion with no major adverse events from midlines. ${ }^{10}$ Importantly, these studies all represent single-centre experiences. Little is known about real-world use and variation of outcomes related to midlines within and across hospitals. Given this gap, we conducted a pilot study to examine use, variation and outcomes related to midline catheters in hospitals across the state of Michigan.

\section{METHODS}

\section{Study setting and design}

This multicentre, prospective cohort study was conducted using data from a 48-hospital collaborative quality initiative supported by Blue Cross Blue Shield of Michigan and Blue Care Network. The design and setting of this consortium have been previously described. ${ }^{11-13}$ In brief, adult patients admitted to a general medicine ward or intensive care unit of a participating hospital who received a midline device as part of their clinical care were eligible for inclusion. Patients (1) under the age of 18, (2) pregnant, (3) admitted to a non-medicine service (eg, general surgery) or (4) admitted under observation status were excluded.

A pilot study to evaluate midline catheter use and outcomes at participating hospitals was launched in July 2017. Twelve hospitals that represented diverse geographical regions, varying bed size and varying volume of midline utilisation were selected to participate in the pilot. At each hospital, dedicated, trained medical record abstractors used a defined protocol to collect clinical data directly from the medical records of patients who received midlines. Based on finite resources for abstractors and the time required to collect data from each case, hospitals were asked to collect data on six midline cases that met the eligibility criteria every 2 weeks (abstraction cycle). Hospitals used a combination of electronic medical records (insertion notes and billing data) and records from inserters (interventional radiology and vascular access teams) to identify eligible patients within each abstraction cycle. Inclusion and exclusion criteria were then applied to each patient on this list and data on the first six eligible cases were collected. To prevent sampling bias based on the day of placement, abstractors sorted cases by date of insertion or medical record number, and the first midline placed each day was abstracted. This process of identifying and abstracting data from eligible patients appropriate was repeated every 2 weeks (see online supplementary Appendix for protocol). All patients were prospectively followed until midline removal, death or at 30 days (whichever occurred first). If a patient was discharged with a midline in place or if the status of the midline was not clear at the time of discharge, patients were contacted by phone to determine the presence of the device and complications.

\section{Covariates and definitions}

Midlines were defined as vascular access devices inserted in the veins of the upper extremity that terminated in the brachial, basilic or cephalic veins of the arm at or near the axillary line. Thus, short peripheral intravenous catheters and PICCs were excluded from this pilot. However, a PICC placed at or trimmed to a 'midline' position was included. Medical diagnoses, history, physical findings, medications and baseline laboratory values at the time of midline placement were abstracted directly from patient medical records. Data regarding midline insertion (eg, indication for use, vein/arm of insertion, gauge, lumens) were obtained directly from the vascular nursing or interventional radiology note or from the physician order for midline placement. In cases where multiple indications for midline use were listed, the primary indication for midline insertion was selected according to a predefined hierarchical structure (eg, difficult intravenous access 1, antibiotics 2, intravenous fluids 3, unknown 4).

\section{Clinical outcomes}

The main outcome of interest included the proportion of midlines that experienced a major or minor complication. Major complications were defined as bloodstream infection related to the midline catheter (captured using a standardised definition from the National Healthcare Safety Network), ${ }^{14}$ symptomatic upper-extremity deep vein thrombosis (DVT) (defined as performance of a compression or duplex ultrasound for arm symptoms with visible thrombus or non-compressibility of the vein) and catheter occlusion (defined as inability to aspirate or flush, or 'sluggish', 'slow' and 'poor' flow documented in the medical record). Minor complications were defined using standardised definitions and included mechanical events (accidental dislodgement, leaking from the exit site and thrombophlebitis), infiltration of infusate outside of a vein and superficial thrombophlebitis. ${ }^{2}$ For this analysis, data from patients enrolled during the pilot period between January 2017 and March 2018 were included.

\section{Statistical analyses}

Indication for midline insertion, dwell time, device characteristics and complications were tabulated using descriptive statistics. Comparisons between minor and major complications and by number of device lumens were made using $\chi^{2}$ tests. In addition to device complications, the rates of midline use (midline utilisation rate) for each hospital were estimated by expressing the proportion of midlines placed in adult non-surgical patients to the total number of non-surgical adult discharges during the same period. This statistic was useful to assess how often midlines were placed relative 
Table 1 Pilot hospital characteristics and midline utilisation data, January 2017-March 2018

\begin{tabular}{|c|c|c|c|c|c|c|c|c|c|}
\hline \multirow[b]{2}{*}{$\begin{array}{l}\text { Site number } \\
\text { hospital ID }\end{array}$} & \multicolumn{4}{|c|}{ Facility characteristics } & \multicolumn{5}{|c|}{ Midline data } \\
\hline & $\begin{array}{l}\text { Total } \\
\text { beds (n) }\end{array}$ & $\begin{array}{l}\text { Total } \\
\text { discharges } \\
\text { (n) }\end{array}$ & $\begin{array}{l}\text { Average } \\
\text { LOS, days }\end{array}$ & $\begin{array}{l}\text { Average } \\
\text { Charlson-Deyo } \\
\text { Score }\end{array}$ & $\begin{array}{l}\text { Midlines } \\
\text { placed } \\
(\mathrm{n})\end{array}$ & $\begin{array}{l}\text { Total } \\
\text { midlines } \\
\text { abstracted, } \\
\text { n (\%) }\end{array}$ & $\begin{array}{l}\text { Hospital } \\
\text { Midline } \\
\text { utilisation } \\
\text { rate } \\
(\%) \\
\end{array}$ & $\begin{array}{l}\text { Median } \\
\text { dwell } \\
\text { time, days } \\
\text { (IQR) }\end{array}$ & $\begin{array}{l}\text { Dwell } \\
\text { time }<5 \\
\text { days }(\%)\end{array}$ \\
\hline Site 1 & 330 & 10623 & 4.5 & 5.80 & 1248 & $150(12.0)$ & 11.75 & $4(2-6)$ & 67.3 \\
\hline Site 2 & 584 & 16657 & 3.6 & 4.76 & 1837 & $91(5.0)$ & 11.03 & $5(3-10)$ & 59.3 \\
\hline Site 3 & 443 & 10464 & 5.0 & 4.91 & 756 & $88(11.6)$ & 7.22 & $6(3-30)$ & 43.2 \\
\hline Site 4 & 404 & 16503 & 4.2 & 6.69 & 1740 & $98(5.6)$ & 10.54 & $5(2-9)$ & 57.1 \\
\hline Site 5 & 317 & 10320 & 3.9 & 6.37 & 100 & $59(59.0)$ & 0.97 & $12(8-30)$ & 17.0 \\
\hline Site 6 & 305 & 2760 & 4.5 & 6.15 & 190 & $137(72.1)$ & 6.88 & $4(2-9)$ & 56.9 \\
\hline Site 7 & 1070 & 35051 & 5.1 & 5.57 & 1008 & $90(8.9)$ & 2.88 & $9(5-14)$ & 30.0 \\
\hline Site 8 & 378 & 14827 & 4.5 & 5.09 & 171 & $77(45.0)$ & 1.15 & $7(3-14)$ & 40.3 \\
\hline Site 9 & 877 & 21205 & 4.9 & 5.45 & 474 & 93 (19.6) & 2.23 & $5(2-9)$ & 57.0 \\
\hline Site 10 & 273 & 9977 & 4.1 & 4.55 & 1289 & $67(5.2)$ & 12.92 & $5(3-9)$ & 52.2 \\
\hline Site 11 & 400 & 12430 & 4.8 & 6.03 & 746 & $95(12.7)$ & 6.00 & $7(4-18)$ & 40.0 \\
\hline Site 12 & 310 & 5600 & 2.5 & 5.14 & 359 & $116(32.3)$ & 6.41 & $7(4-25)$ & 41.4 \\
\hline All sites & 5691 & 166417 & 4.3 & 5.58 & 9918 & $1161(11.7)$ & 7.04 & $6(3-12)$ & 49.0 \\
\hline
\end{tabular}

LOS refers to the average length of stay for patients admitted to that hospital across all services.

Midline utilisation rate=total number of midlines placed per year in adult non-surgical patients/number of adult non-surgical patients discharged per year

to patient volume. Associations between patient factors (severity of illness as defined by the Charlson-Deyo Score), hospital factors (bed size, hospital volume) and rates of midline utilisation by site were assessed using Pearson's correlation. All statistical tests were two-sided, with $\mathrm{p}<0.05$ considered statistically significant. All analyses were conducted using Stata MP/SE V.15.

\section{RESULTS}

Complete data on 1161 midlines placed in 12 hospitals were available and included in this analysis. The available sample of midlines represented $5 \%-72 \%$ of all devices placed in these hospitals during the study period (table 1). Most midlines were placed in general ward settings ( $\mathrm{n}=822,70.8 \%)$; however, $24.2 \%$ $(\mathrm{n}=281)$ were placed in critical care units and $5.0 \%$ $(\mathrm{n}=58)$ in 'other' settings (eg, emergency room). The majority of midlines were placed by vascular access nurses at the patients bedside $(\mathrm{n}=1036,89.2 \%)$. The documented indication for $61.4 \%$ of midlines $(n=713)$ was difficult intravenous access; however, intravenous antibiotics represented approximately one-third of all placement indications $(\mathrm{n}=318,27.4 \%)$. Consultation with infectious diseases for placement of midlines for intravenous antibiotics occurred for less than half of all midlines placed for this indication (46.2\% [12.7\% of all midlines]), suggesting a potential opportunity for device and antibiotic stewardship. Almost a quarter $(23.9 \%, n=278)$ of patients had more than one documented indication for midline placement, while the indication for midline insertion was unknown (ie, none documented in medical records or procedure notes) in $16.1 \%$ of cases.
The right arm was more often used for midline placement $(\mathrm{n}=597,51.4 \%)$. The basilic $(\mathrm{n}=487$, $42.0 \%)$ and brachial $(n=469,40.4 \%)$ veins were most commonly used for insertion. Single-lumen devices represented almost half of all midlines (46.0\%). Ultrasound guidance was documented for placement in $80.6 \%$ of midline devices.

The median dwell time of midlines across hospitals was 6 days (IQR: $3-12$ days), with almost half ( $n=569$, $49.0 \%)$ removed within 5 days of insertion. The most common reasons for removal within 5 days $(67.8 \%$ of 5-day midline dwells) were completion of therapy and patient discharge. A total of $13.5 \%$ of midlines remained in place beyond 30 days (figure 1a). Most patients $(n=994,85.6 \%)$ who received a midline did not have a PICC or central venous catheter placed within the past 3 months, suggesting that midlines were not used as 'step-down' device from more invasive lines. Informed consent for midline catheter placement was obtained in 36.2\% $(n=420)$ of patients.

\section{Device complications}

A major or minor complication occurred in 10.3\% of midline insertions (table 2). Minor complications accounted for the majority of adverse events $(66.7 \%$ of all complications). In order of frequency, minor complications included accidental dislodgement (3.8\%, $\mathrm{n}=44)$, leaking from the exit site $(2.2 \%, \mathrm{n}=26)$, catheter infiltration $(n=7,0.6 \%)$ and superficial thrombophlebitis $(n=4,0.3 \%)$. Although major complications accounted for a third of all adverse events, the most frequent major complication was catheter occlusion $(2.2 \%, n=26)$. Rates of symptomatic upper-extremity DVT and bloodstream infection were low across 
A Median Dwell Days by Site

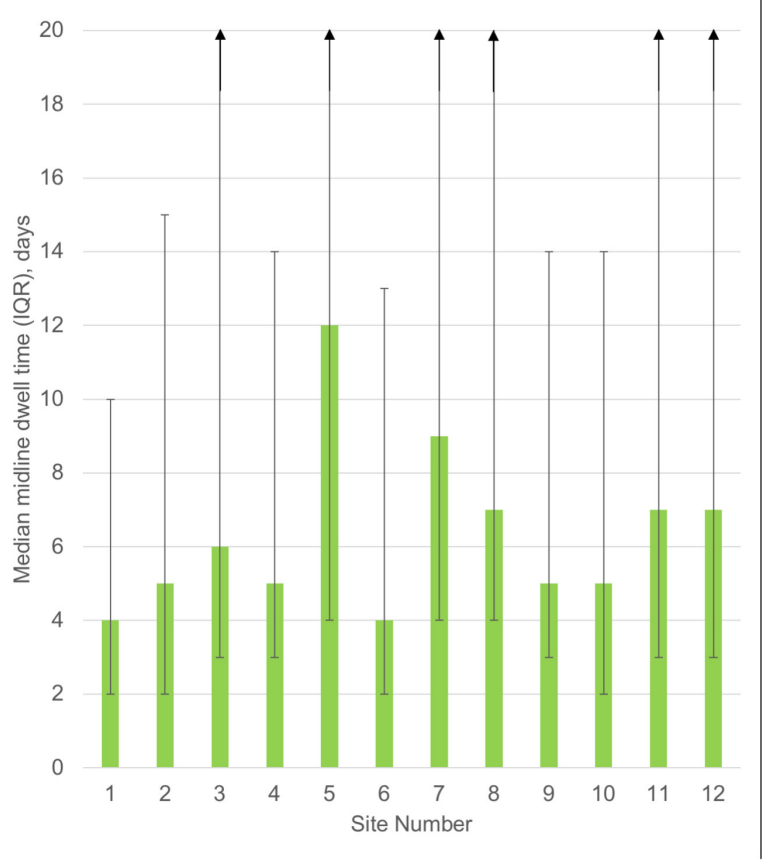

B Indication by Site

160

140

120

80

60

40

20

0

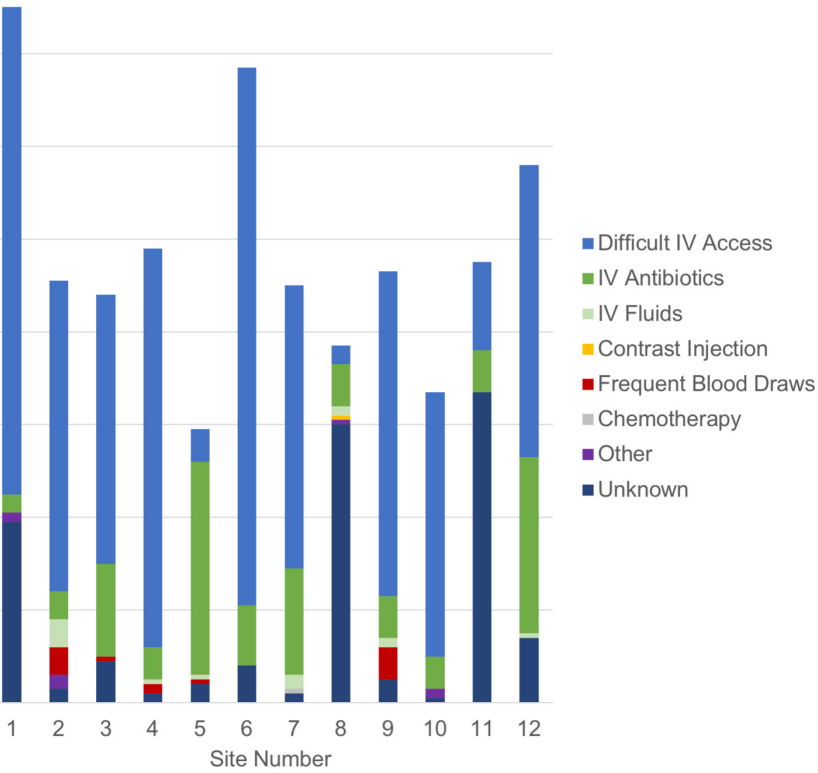

Figure 1 (A) Median midline dwell time and (B) indication (by site). The vertical bars in Panel (A) show range of catheter dwell (in days).

patients $(1.4 \%[n=16]$ and $0.3 \%[n=4]$, respectively). Rates of midline removal were similar among patients who developed major or minor complications $(53.8 \%$ vs $52.5 \%, \mathrm{p}=0.90)$. With respect to the number of lumens, single-lumen midline catheters appeared to have lower rates of complications than multilumen devices (single-lumen $=9.0 \%, \quad$ multilumen $=12.8 \%$; $\mathrm{p}=0.23)$. Among patients with midlines placed for $<5$ days, $12.7 \%(\mathrm{n}=72)$ experienced a complication and $7.2 \%(n=41)$ had their device removed due to this complication.

\section{Variation in use and outcomes of midlines across hospitals}

The absolute volume of midline use varied across hospitals, ranging from 100 to 1837 devices, with corresponding midline utilisation rates of $0.97 \%-12.92 \%$ $(\mathrm{p}<0.001)$. Overall, the sample of devices in this study represented $5 \%-72 \%$ of all midlines placed at individual hospitals; thus, some hospitals used very few midlines, while others used more. Variation in midline utilisation rate across hospitals was not associated with the severity of patient illness by Charlson-Deyo Score $(\mathrm{r}=-0.20, \mathrm{p}=0.540)$, or by hospital-level factors such as patient volume $(\mathrm{r}=-0.30, \mathrm{p}=0.337)$ or bed size $(-0.37, p=0.231)$. Additionally, dwell time and indications for midline use varied across hospitals (figure 1A,B). For example, placement of midlines for the indication of difficult venous access varied from $5.2 \%$ to $87.8 \%$ across sites $(p<0.001)$. Similarly, the frequency of midline complications varied from $3.4 \%$ to $16.7 \%$ across hospitals $(p=0.07)$ (table 2$)$. Notably, patterns of complications also differed across hospitals.
For example, three hospitals reported no upper-extremity DVT events from midlines. Among the nine hospitals that did report DVT, incidence varied from $0.7 \%$ to $3.3 \%$ of patients who received midlines. Similarly, although accidental dislodgement was the most prevalent minor complication, rates for this event ranged from $1.1 \%$ to $6.1 \%$ across sites. No differences in rates of complications by device manufacturer were observed $(\mathrm{p}=0.68)$.

\section{DISCUSSION}

As hospitals seek to improve appropriate use of PICCs to avoid adverse events such as DVT and financial penalties associated with CLABSI, interest in use of midline devices has emerged. ${ }^{15}$ Despite growing use of these devices, a paucity of epidemiological data regarding patterns of use and infectious and non-infectious outcomes exists. In this multihospital pilot study spanning twelve sites and 1161 midline insertions, we found that midlines were most often placed for the indications of difficult intravenous access and antibiotic therapy. Almost half of midlines placed were removed within 5 days of insertion after having met the indication they were placed for, with few of these shorter dwells (7\%) removed for complications. Among patients who did experience a complication, minor complications were more common than major events. However, both types of complications often required midline removal, suggesting that minor complications are not inconsequential from a vascular access or patient perspective. Like prior work with PICCs, ${ }^{12}$ we found substantial variation in patterns of use and outcomes from midlines across hospitals. 


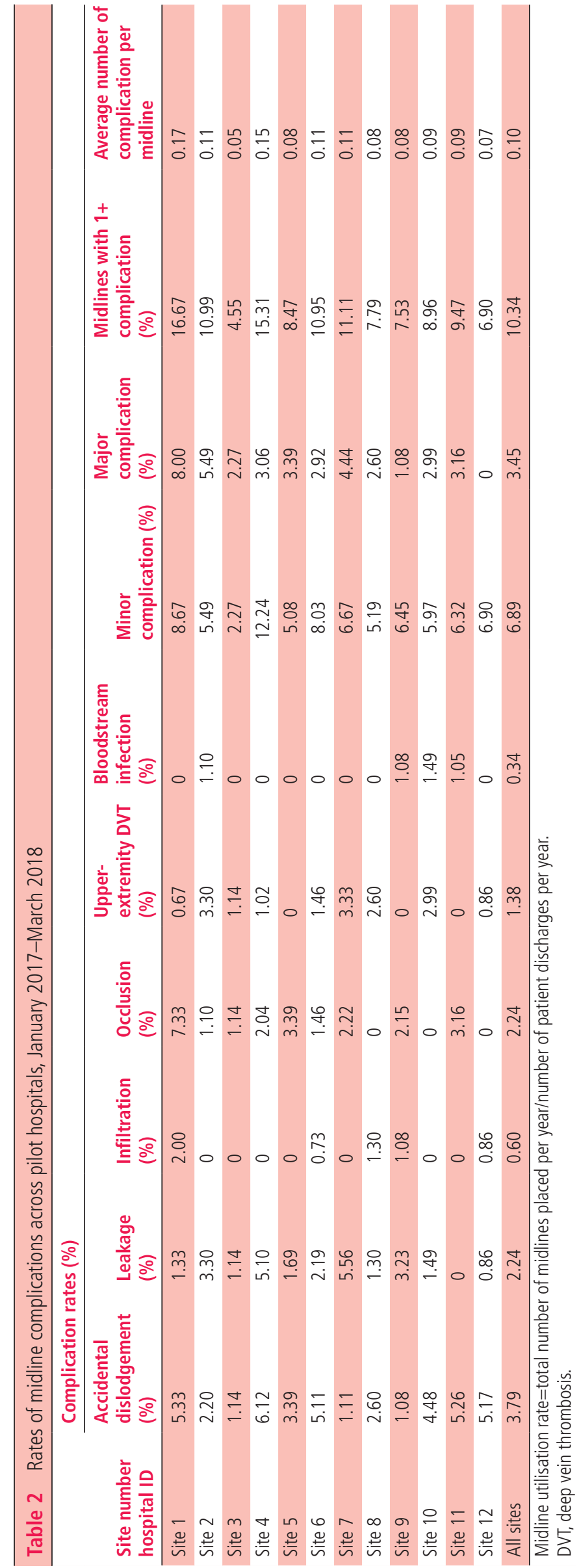

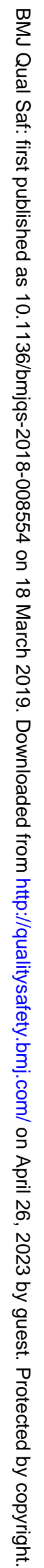


Collectively, these data suggest that guidance to inform and improve use of midlines is needed in order to ensure patient safety.

Although rates of complications with midlines were low, significant variation in both the nature and types of adverse events across hospitals was observed. Importantly, midlines appear to be more often associated with minor than major complications, with low rates of DVT and bloodstream infection noted across hospitals. These observations are congruent with recent studies and suggest that midlines might be a 'safer' alternative to PICCs with respect to these outcomes. ${ }^{89}$ Given their lower rate of potentially lethal events such as DVT and CLABSI, midlines appear to have an important place for hospitalised patients, especially for those who need short-term venous access. Compared with prior studies that found substantially greater rates of complications, ${ }^{16}$ our data suggest that the complication profile of midlines has improved perhaps due to newer catheter materials or ultrasound to guide insertion.

Despite these assurances, when and how midlines should be used to minimise risk and ensure benefit remain an important question. The Michigan Appropriateness Guide for Intravenous Catheters (MAGIC) provides criteria for when use of a midline versus a PICC is appropriate for hospitalised and critically ill patients and patients with cancer. ${ }^{17} 18$ Additionally, MAGIC provides guidance on what types of infusions are appropriate for a midline device-a key component of mitigating harm from these devices. As has been the case with PICCs, ${ }^{10}$ using MAGIC as a framework to understand and improve midline appropriateness and patient outcomes may be valuable in improving patient safety.

We observed that most midlines were placed for the indication of difficult venous access, suggesting that hospitals are turning to this device when attempts to cannulate peripheral veins fail. As midlines are longer in length, placed under sterile conditions and usually placed by ultrasound, they reach the deeper veins of the arm to secure more durable access. However, variation in indication for placement and rates of midline dwell were observed across hospitals. Thus, how best to use, insert and maintain midlines to ensure catheter dwell matches clinical need remains an important outstanding question. Studies that focus on insertion parameters related to midlines, including use of ultrasound, length of catheter within the vein and catheter-to-vein ratio, are thus needed as they may help prolong dwell. Similarly, identifying optimal care and maintenance techniques including what should be adminstered via a peripheral device such as a midline, volume, type and frequency of flush and optimal securement strategies to prevent dislodgement also appear necessary. ${ }^{19}$ These considerations represent important questions for future research and are needed to fully realise the potential of these devices.
Our study has limitations. First, we collected data from the electronic medical records of hospitalised patients via trained data abstractors; data or events that were not documented or occurred after hospitalisation and were not reported by patients during phone conversations may be missed using this method. Second, we sampled patients at hospitals in order to understand patterns and outcome of midline use. Differences between our study sample and those from the larger population of patients who received midlines may affect our findings. Third, while we did not find an association between hospital-level and patient-level factors related to midline use or outcome, it is possible that we were underpowered for this effect given the pilot nature of this study. Fourth, complications with midlines (including major complications such as infection and DVT) may be under-reported or under-recognised as these are peripheral intravenous devices. Furthermore, variability in DVT rates between hospitals may also reflect variability in diagnostic testing thresholds, which we were unable to assess. However, our use of medical record documentation (including laboratory and imaging data) rather than administratively coded data helps to mitigate this risk. Finally, outcomes from midlines may vary based on materials, catheter design, insertion techniques, and infusates which we did not collect in this pilot study. Future iterations of this work will seek to incorporate these aspects.

Our study also has important strengths. To our knowledge, this is the first and largest multihospital study to date to examine indications, patterns of use and outcomes for midline catheters. As use of these devices expands, understanding best practices through epidemiological studies such as ours will become more necessary. Second, we report several new findings including the fact that the number of midline lumens appears associated with the rates of complications. These findings have important implications for clinical decision-making and patient safety. Third, substantial variation in hospital event rates related to midline devices appears to exist. Using a conceptual framework of patient, provider and device factors to understand complications thus appears to be just as important for midlines as it was for PICCs. ${ }^{20}$ Future studies should now begin to examine these factors and consider strategies through which to prolong midline dwell so as to ensure best outcomes.

In conclusion, substantial variation in the rates of use and outcomes from midline catheters were observed in this multihospital study. Given lower risk of major complications, we suspect the use of midline catheters over PICCs will continue to expand. Studies to better define appropriate indications for use, optimal insertion technique, and best care and maintenance practices to ensure longevity of these devices are needed to ensure patient safety. 
Author affiliations

${ }^{1}$ The Division of Hospital Medicine, Department of Internal Medicine, University of Michigan Medical School, Ann Arbor, Michigan, USA

${ }^{2}$ Patient Safety Enhancement Program and Center for Clinical Management Research, VA Ann Arbor Health Care System, Ann Arbor, Michigan, USA

${ }^{3}$ The Michigan Hospital Medicine Safety Consortium, Ann Arbor, Michigan, USA

${ }^{4}$ Department of Internal Medicine, Henry Ford Health System, Detroit, Michigan, USA

${ }^{5}$ Department of Internal Medicine, Beaumont Hospital, Dearborn, Michigan, USA

${ }^{6}$ St. Josephs Health Center, Ypsilanti, Michigan, USA

${ }^{7}$ The Division of General Medicine, Department of Internal Medicine, University of Michigan Medical School, Ann Arbor, Michigan, USA

Contributors All authors participated equally in the design, conception, writing, analysis, drafting and final approval of the paper. VC and AS had access to the data and vouch for the integrity of the findings.

Funding Support for the Michigan Hospital Medicine Safety (HMS) Consortium is provided by Blue Cross and Blue Shield of Michigan (BCBSM) and Blue Care Network as part of the BCBSM Value Partnerships program. Although Blue Cross Blue Shield of Michigan and HMS work collaboratively, the opinions, beliefs and viewpoints expressed by the author do not necessarily reflect the opinions, beliefs and viewpoints of BCBSM or any of its employees.

Competing interests None declared.

Patient consent for publication Not required.

Ethics approval Because the purpose of the consortium is to measure and improve the quality of existing care practices, the project received a 'Not Regulated' status from the Institutional Review Board at our hospital.

Provenance and peer review Not commissioned; externally peer reviewed.

Open access This is an open access article distributed in accordance with the Creative Commons Attribution Non Commercial (CC BY-NC 4.0) license, which permits others to distribute, remix, adapt, build upon this work noncommercially, and license their derivative works on different terms, provided the original work is properly cited, appropriate credit is given, any changes made indicated, and the use is noncommercial. See: http://creativecommons.org/licenses/by-nc/4. $0 /$.

\section{REFERENCES}

1 Anderson NR. Midline catheters: the middle ground of intravenous therapy administration. J Infus Nurs 2004;27:313-21.

2 Infusion Nurses Society. Infusion nursing standards of practice, 2016. Available: http://wwwins1org/ Defaultaspx? TabID $=251$ \&productId $=113266$ [Accessed 12 Oct 2018].

3 Moureau N, Sigl G, Hill M. How to establish an effective midline program: a case study of 2 hospitals. J Assoc Vascular Access 2015;20:179-88.

4 Dawson RL. Midline catheters: an essential tool in CLABSI reduction. infection control today. Available: https://www.infe ctioncontroltoday.com/clabsi/midline-catheters-essential-toolclabsi-reduction [Accessed 24 Jun 2018].

5 Adams DZ, Little A, Vinsant C, et al. The midline catheter: a clinical review. J Emerg Med 2016;51:252-8.

6 Paje D, Conlon A, Kaatz S, et al. Patterns and predictors of short-term peripherally inserted central catheter use: a multicenter prospective cohort study. J Hosp Med 2018;13:76-82.

7 Gibson C, Connolly BL, Moineddin R, et al. Peripherally inserted central catheters: use at a tertiary care pediatric center. J Vasc Interv Radiol 2013;24:1323-31.

8 Campagna S, Gonella S, Zerla PA, et al. The risk of adverse events related to Extended-Dwell peripheral intravenous access. Infect Control Hosp Epidemiol 2018;39:1-3.

9 Xu T, Kingsley L, DiNucci S, et al. Safety and utilization of peripherally inserted central catheters versus midline catheters at a large academic medical center. Am J Infect Control 2016;44:1458-61.

10 Swaminathan L, Flanders S, Rogers M, et al. Improving PICC use and outcomes in hospitalised patients: an interrupted time series study using magic criteria. BMJ Qual Saf 2018;27:271-8.

11 Smith SN, Moureau N, Vaughn VM, et al. Patterns and predictors of peripherally inserted central catheter occlusion: the 3P-O study. J Vasc Interv Radiol 2017;28:749-56.

12 Chopra V, Smith S, Swaminathan L, et al. Variations in peripherally inserted central catheter use and outcomes in Michigan hospitals. JAMA Intern Med 2016;176:548-51.

13 Chopra V, Kaatz S, Grant P, et al. Risk of venous thromboembolism following peripherally inserted central catheter exchange: an analysis of 23,000 hospitalized patients. Am J Med 2018;131:651-60.

14 O'Grady NP, Alexander M, Burns LA, et al. Guidelines for the prevention of intravascular catheter-related infections. Clin Infect Dis 2011;52:e162-93.

15 Zamanian K, Collins S. New appropriate use guidelines and their impact on the U.S. vascular access device market. Medical Devices Online 2016.

16 Goetz AM, Miller J, Wagener MM, et al. Complications related to intravenous midline catheter usage. A 2-year study. J Intraven Nurs 1998;21:76-80.

17 Chopra V, Flanders SA, Saint S, et al. The Michigan appropriateness guide for intravenous catheters (magic): results from a Multispecialty panel using the RAND/UCLA appropriateness method. Ann Intern Med 2015;163(6 Suppl):S1-40.

18 Moureau N, Chopra V. Indications for peripheral, midline and central catheters: summary of the magic recommendations. $\mathrm{Br}$ J Nurs 2016;25:S15-24.

19 Ventura R, O'Loughlin C, Vavrik B. Clinical evaluation of a securement device used on midline catheters. Br J Nurs 2016;25:S16-22.

20 Chopra V, Anand S, Krein SL, et al. Bloodstream infection, venous thrombosis, and peripherally inserted central catheters: reappraising the evidence. Am J Med 2012;125:733-41. 